\title{
Philosophiques
}

\section{Identité procédurale ou substantielle?}

\section{Autour du rapport identité-altérité dans le débat libéraux-communautariens}

\section{Soumaya Mestiri}

Volume 35, numéro 2, automne 2008

URI : https://id.erudit.org/iderudit/000436ar

DOI : https://doi.org/10.7202/000436ar

Aller au sommaire du numéro

\section{Éditeur(s)}

Société de philosophie du Québec

ISSN

0316-2923 (imprimé)

1492-1391 (numérique)

Découvrir la revue

Citer cet article

Mestiri, S. (2008). Identité procédurale ou substantielle? Autour du rapport identité-altérité dans le débat libéraux-communautariens. Philosophiques, 35(2), 419-450. https://doi.org/10.7202/000436ar

\section{Résumé de l'article}

La question de l'identité n'est pas nouvelle, mais c'est sans nul doute avec l'avènement du débat libéraux-communautariens consécutif à la parution de Théorie de la justice de Rawls que l'acuité et la portée du problème identitaire transparaît nettement dans les travaux théoriques des uns et des autres. Aux libéraux, censés nous présenter une version procédurale de l'identité, s'opposent les communautariens et leur vision de la personne profondément ancrée dans des valeurs qu'elle ne pourrait renier sans se perdre à jamais elle-même.

Pour autant, l'intuition qui guide ce travail est qu'un certain libéralisme peut tout à fait parvenir à proposer une vision conséquente de l'identité. Le présent propos s'attache à reconsidérer les termes de cette confrontation en donnant essentiellement la parole à trois philosophes (les communautariens Taylor et Walzer, et le libéral Rawls), sans pour autant s'interdire d'autres références moins impliquées dans ce débat (Tamir). 


\title{
Identité procédurale ou substantielle?
}

\section{Autour du rapport identité-altérité dans le débat libéraux-communautariens}

\author{
SOUMAYA MESTIRI \\ Université de Tunis \\ mestirisoumaya@yahoo.fr
}

\begin{abstract}
RÉSUMÉ. - La question de l'identité n'est pas nouvelle, mais c'est sans nul doute avec l'avènement du débat libéraux-communautariens consécutif à la parution de Théorie de la justice de Rawls que l'acuité et la portée du problème identitaire transparaît nettement dans les travaux théoriques des uns et des autres. Aux libéraux, censés nous présenter une version procédurale de l'identité, s'opposent les communautariens et leur vision de la personne profondément ancrée dans des valeurs qu'elle ne pourrait renier sans se perdre à jamais elle-même.

Pour autant, l'intuition qui guide ce travail est qu'un certain libéralisme peut tout à fait parvenir à proposer une vision conséquente de l'identité. Le présent propos s'attache à reconsidérer les termes de cette confrontation en donnant essentiellement la parole à trois philosophes (les communautariens Taylor et Walzer, et le libéral Rawls), sans pour autant s'interdire d'autres références moins impliquées dans ce débat (Tamir).
\end{abstract}

\begin{abstract}
The question of identity is surely not a new one. However, the rise of the liberal-communautarian debate as a consequence of the publication of $A$ theory of justice gave it a real acuity. Indeed, liberals, with their so-called procedural vision of identity, conflict with communautarians and their understanding of the person as genuinely embedded in a set of values she couldn't repudiate without disappearing herself.
\end{abstract}

This study works on an adjustment of this Manichean setting of the problem, by establishing a dialogue between three philosophers : Taylor and Walzer, as members of the communautarian team, and Rawls, as the liberal opponent; this, however, won't prevent us from working on authors who are not directly involved in this debate (Tamir). The main idea of this paper is that a particular liberalism is able to propose a vision of identity which takes seriously the most communautarian criticisms, so that the traditional gap between the two doctrines becomes deeply meaningless.

Des études récentes d'anthropologie politique montrent que le phénomène social ne saurait se réduire, comme c'était traditionnellement le cas, à deux sphères d'appartenance, la sphère inclusive, celle de la sécurité, de l'acquis et de l'échange, et la sphère exclusive, celle de la fragilité, de la déshérence et de la 
perte de repères. De fait, à côté de ces deux champs classiques imperméables l'un à l'autre, ou, plus exactement, au cœur de l'espace laissé libre entre eux deux, se crée une troisième sphère qui fait en quelque sorte le lien entre les deux premières. C'est la sphère de l'acclusion, de ceux qui ne sont ni inclus ni exclus, qui tendent à la fois vers l'un et vers l'autre, en mouvement permanent, comme si au fond il n'y avait pas de rupture réelle entre les deux espaces traditionnels, mais qu'un pont était jeté entre eux, charriant ceux qui évoluent en marge des normes traditionnelles sans pour autant que cette errance ait quoi que ce soit de définitif. Pierre Bouvier résume parfaitement cela lorsqu'il explique que :

L'acclusion est la phase pivot résultant de la séparation et de la désagrégation du statut antérieur, celle d'inclus. Elle donne à voir une situation intermédiaire de liminalité [...] L'individu flotte dans un entre-deux [...], circulant, dans un mouvement pendulaire de durée plus ou moins longue, entre l'une ou l'autre des oppositions que sont l'inclusion ou l'exclusion (Bouvier, 2005, p. 256).

Cette lecture du phénomène social fournit un certain nombre d'éléments en faveur de l'idée selon laquelle personne, en réalité, ne peut se considérer aujourd'hui comme un inclus. De fait, notre appartenance à tel ou tel groupe n'est jamais définitive, et nous passons de l'une à l'autre au gré de notre évolution personnelle, de sorte qu'au fond un instantané de notre quotidien montrerait que nous nous mouvons dans l'acclusif, toujours en instance d'entrée ou de sortie.

Le présent propos s'intéresse aux conséquences de l'introduction d'une telle catégorie dans la philosophe politique contemporaine et, plus particulièrement, au sein du traditionnel clivage entre libéraux et communautariens qui la domine. Les griefs que les seconds adressent aux premiers, notamment au regard de leur atomisme réducteur et des conséquences négatives qui en découlent, demeurent-ils toujours pertinents une fois que l'on a mis au jour la positivité essentielle de cette mobilité structurelle ${ }^{1}$ ?

Ces interrogations nous permettront de poser les jalons de l'existence d'une conception forte de l'altérité au sein d'un certain libéralisme procédural, celui de Rawls. Nous partirons de l'alternative taylorienne. De fait, selon le philosophe canadien, un modèle cohérent existe qui vient corriger l'aberration représentée par le concept même de moi désengagé : le modèle associatif à la Humboldt, bâti tout à la fois sur la reconnaissance de l'autre et sur une réalité essentielle, à savoir que nous évoluons tous dans une interdépendance structurelle avec nos semblables (I). L'image que donne Taylor du libéralisme

1. Forst (1993) propose une variation intéressante de l'idée d'identité mobile, à savoir l'identité complexe. La personne n'est ni le soi libéral désencombré incriminé par les communautariens ni une somme de caractéristiques et de valeurs données une fois pour toutes, mais se conçoit bien plutôt comme une construction à quadruple niveau: éthique, légal, civique et moral, p. 303-305. 
procédural, quoique globalement juste ${ }^{2}$, apparait toutefois caricaturale et biaisée s'agissant du cas particulier de la théorie rawlsienne de la justice comme équité. Nous montrerons comment cette dernière est informée par une idée centrale, celle d'union sociale, dont tout l'intérêt est de recenser les avantages du modèle alternatif taylorien (II). Reste que les communautariens dénoncent la qualité des liens tissés au sein de l'union sociale : ce sont des liens ponctuels, qui ne durent pas, car nos appartenances sont par définition provisoires et nos attaches appelées à se dissoudre. C'est ici que se manifeste l'importance de la notion d'acclusion: si nous flottons dans un espace-flux mobile et changeant, si cette mobilité est ce qui définit structurellement notre identité, alors comment stigmatiser cette priorité du moi sur ses fins? Apparaît dès lors l'idée que ce qui gêne n'est pas tant le côté structurel ainsi envisagé, mais ce qui vient le fonder. En d'autres termes, l'ennui réside dans le fait que cette mobilité est une somme de sympathies, de loyautés et d'engagements purement volontaires: les liens que nous créons les uns avec les autres, quels qu'ils soient, témoignent exclusivement de notre capacité à juger en connaissance de cause et à choisir. Telle est l'objection que Walzer fait à Rawls. Nous en exposerons les limites, notamment au regard de la conception rawlsienne de l'association (III). Nous examinerons ensuite une critique fondamentale que le communautarisme adresse au libéralisme procédural: en raison de sa neutralité à l'égard des conceptions du bien, ce dernier disqualifierait d'emblée la question de l'identité collective. Sa vision de l'État et des institutions serait clairement instrumentale, bien loin d'un telos fédérateur autour duquel se rejoindraient tous les citoyens. À rebours de cette idée reçue, et notamment à travers une analyse confrontant la figure du resquilleur à l'individu rawlsien, nous donnerons à voir une préoccupation centrale de la théorie de la justice comme équité, à savoir l'engagement civique protéiforme (IV). Mais il y a plus : l'identité promue par Rawls n'est pas simplement civique, ainsi que le donnent à voir les concepts de peuple et de nation tels qu'il les développe dans le Droit des peuples: sa société bien ordonnée aurait ainsi toutes les apparences d'une «communauté nationale» $(\mathrm{V})$. Une question demeure toutefois en suspens : le libéralisme procédural rawlsien prend-il vraiment l'autre au sérieux? Sans indifférence ni mépris, nous montrerons que les subtilités de la conception de

2. Une critique fort connue de l'absence de la figure de l'autre dans la théorie de la justice comme équité est celle développée par Habermas dans le débat célèbre qui l'a opposé à Rawls. Selon le philosophe allemand, la position originelle est monologique : il n'y a pas de véritable dialogue entre les personnes derrière le voile d'ignorance, et l'unanimité autour du choix des deux principes de justice en est la preuve irréfutable. Tout se passe comme si la procédure qui préside à la sélection de ces derniers n'était en réalité suivie que par un seul et unique individu imposant les normes qu'il juge justes sans prendre la peine (et le risque) de les soumettre à ses semblables. Rawls a certes répondu à cette critique en affirmant que la position originelle s'apparentait à un " omnilogue ", un dialogue de chacun avec tous et de tous avec chacun. Nous pensons malgré tout qu'Habermas a raison pour ce cas particulier de la position originelle; mais nous aimerions montrer que la monologie ou négligence de la figure de l'autre n'est pas pour autant généralisable à l'ensemble de la théorie rawlsienne, tant s'en faut. Voir DJP, p. 65. 
la tolérance défendue par Rawls ont de quoi dérouter les multiculturalistes les plus engagés et les apôtres de la différence les plus fervents (VI).

\section{Quelle politique de la différence?}

Il est indéniable que le libéralisme procédural est un modèle valable et qu'il bénéficie d'un "grand avantage » puisqu'il repose sur l'idée que l'égalité de traitement suppose une absence de prise en considération des particularités individuelles de chacun - un postulat pour le moins respectable (Taylor, 1998, p. 213).Toutefois, le libéralisme procédural ne saurait être viable dans tous les cas de figure: être aveugle à la différence n'est pas toujours la meilleure façon de rendre justice à chacun ${ }^{3}$.

Taylor propose un modèle de remplacement, susceptible de tenir positivement compte de la différence et pas simplement de la mettre de côté pour bâtir une justice procédurale, c'est-à-dire purement formelle. Dans cette configuration innovante, l'autre n'est pas celui dont je me démarque et en dépit duquel je me construis, mais bien plutôt celui grâce auquel je m'épanouis. La différence m'enrichit comme elle enrichit mes vis-à-vis, dans le sens où ma vie et la leur seront moins pleines, plus étriquées, si nous évoluons indépendamment les uns des autres sans être associés dans une «entreprise commune» (ibid., p. 214). Ériger la différence en valeur, c'est supposer que nous sommes « complémentaires» (ibid.)

Une version intéressante de ce modèle est, note Taylor, celle de Humboldt. Sa conception de la liberté individuelle est fort juste dans la mesure où elle pose que chacun d'entre nous a un intérêt moral à ce que les autres puissent développer pleinement toutes leurs potentialités. S'appuyant sur Herder, le philosophe canadien montre que la métaphore qui traduit le mieux cette complémentarité et ce lien essentiel qui me lient à l'autre est celle de l'orchestre ou du chœur. Tout comme l'harmonie musicale ne se réalise que par des instruments qui jouent à l'unisson des partitions écrites pour eux, tout comme l'harmonie vocale ne se réalise que par des voix qui chantent en rythme des mélodies composées pour elles, de la même manière, les humains s'associent au regard de leurs compétences et de leurs talents (et non sur une base contractuelle et formelle) pour créer l'humanité.

3. L'idée selon laquelle le libéralisme en général (et le libéralisme procédural en particulier) offrent un modèle socio-politique valide, définitivement intégré et assimilé, est affirmée de manière fort pertinente par Michael Walzer: «Le langage des droits individuels — l'association volontaire, le pluralisme, la tolérance, la séparation, la vie privée, la liberté de parole, l'ouverture des carrières au talent, etc. - est tout simplement inévitable. Y en a-t-il parmi nous qui songent sérieusement à y échapper ? ", in Walzer (1997a), p. 68. L'auteur de Sphères de justice insiste sur le fait que si ce modèle a créé, comme le stigmatise la critique communautarienne, une société fortement décomposée et "désencombrée ", c'est à lui, peut-on raisonnablement penser, que reviennent à la fois la responsabilité et le légitimité de sa remise en cause et de son éventuel amendement : «[...] nous pouvons supposer que la politique libérale est la mieux armée pour faire face aux conséquences problématiques de cette décomposition ", ibid., p. 58. 
C'est cette conscience d'une réalisation commune, d'un projet auquel chacun apporte sa pierre, qui manquerait au libéralisme procédural. Celuici «ne nous encourage pas à connaître les points de vue des autres » parce qu'il n'envisage aucunement la possibilité d'une «fusion des horizons» (ibid., p. 215). Pour le dire autrement, le libéralisme considère que nous n'avons rien à gagner des autres puisque notre identité se construit exclusivement sur notre propension à faire des choix.

Cette incapacité foncière du libéralisme procédural à envisager l'autre comme mon complément nécessaire se double d'un refus, celui de comprendre que les doctrines compréhensives des uns et des autres, en tant que sommes d'appartenances, de convictions et de sympathies, ne peuvent purement et simplement être évincées de la sphère publique. Taylor donne à voir cela dans un exemple précis, celui de la prière à l'école. Il examine deux types de réponses négatives que des responsables authentiquement libéraux pourraient opposer à une communauté chrétienne qui défend la possibilité, pour ses plus jeunes fidèles, de pratiquer leur religion dans l'enceinte scolaire. La première fin de non-recevoir insiste sur l'injustice fondamentale qu'il y aurait à autoriser la prière pour les chrétiens, et à la refuser pour « les juifs, les musulmans, les hindous, les bouddhistes » (ibid., p. 217). Dans le second cas, les instances concernées rejettent la requête au nom de certains "principes moraux qui fondent la société libérale et qui sont enracinés dans [la] constitution américaine ", tout en accompagnant leur refus d'un mépris certain pour leurs interlocuteurs : pour ignorer une vérité aussi évidente que celle qui fonde le texte juridique américain le plus important, affirment-elles, « il faut être soit un individu tout à fait primitif, soit un ennemi de [ladite] constitution» (ibid.).

La première réponse "prend au sérieux » les exigences de la communauté chrétienne. De fait, elle ne stigmatise ni la trivialité de ses revendications ni leur fausseté mais y reconnaît l'expression d'un désir sinon légitime, du moins intelligible. Une telle reconnaissance implique qu'un effort a été fait, lequel manifeste la volonté de comprendre ce qui est réellement en jeu pour les membres de cette communauté. À l'inverse, le second type de discours, non seulement coupe d'emblée court à toute conversation mais, plus encore, donne une image «fort peu flatteuse » des fidèles concernés tout en exigeant d'eux d'opérer une conversion totale afin que la prière à l'école «cesse d'être une expression normale de leur mode de vie chrétien » (ibid.). Et Taylor d'ajouter que si les chrétiens doivent reconsidérer leur identité et se faire agnostiques kantiens pour pouvoir bénéficier de l'égalité de traitement et de respect prônée par le libéralisme procédural, nul doute que nous serions alors "plutôt face à une mauvaise plaisanterie qu'à de la philosophie politique digne de ce nom » (ibid., p. 218). Au demeurant, les tentatives libérales pour offrir une théorie politique convaincante du point de vue de son appréhension de l'autre et d'un traitement acceptable de la question identitaire, connaissent toutes un échec cuisant; le second (dernier) Rawls et son tournant politique opéré depuis Libéralisme politique, en est un exemple flagrant. 
La préférence du philosophe canadien, on l'aura bien compris, va donc au modèle associatif à la Humboldt qui donne au respect sa vraie valeur. Respecter l'autre, ce n'est pas lui être indifférent, mais le considérer comme un individu particulier, avec ses attaches, ses idées et ses croyances particulières. Respecter l'autre, c'est donc fondamentalement récuser une certaine interchangeabilité des personnes, ce qui est précisément ce sur quoi se fondent le libéralisme procédural et son formalisme outrancier. Selon le philosophe canadien, la manière dont le modèle associatif humboldtien conçoit l'altérité (et, ce faisant, l'identité personnelle) lui permet d'envisager une société véritablement démocratique dans laquelle l'échange avec les autres, au sein de débats féconds, est une réalité et non un vœu pieux. Telle est la "communauté délibérative » à laquelle Taylor aspire (ibid., p. 220).

Naturellement, ce type d'association implique une forte cohésion entre ses membres, que notre penseur résume sous la forme d'une triade sonnant comme un credo : connaître, écouter, comprendre (ibid.). Ce sont là les conditions sine qua non d'une délibération véritable. La question qui se pose alors est de savoir comment garantir cette cohésion et comment la faire perdurer. Seule la conscience d'une identité collective peut assurer l'engagement des uns vis-à-vis des autres, et réciproquement :

[...] Un État démocratique moderne exige un peuple doté d'une forte identité collective. La démocratie nous oblige à faire preuve de beaucoup plus de solidarité et de bien plus d'engagement les uns à l'égard des autres au sein de notre projet politique commun que ne l'exigeaient les sociétés hiérarchiques et autoritaires d'antan (ibid., p. 221).

Ainsi, l'avantage, si l'on peut dire, du sentiment d'être mû par la même force que mes concitoyens et d'œuvrer dans le même sens qu'eux est que je ne saurais me sentir exclu(e), d'une manière ou d'une autre, de la communauté que nous formons. Mes revendications seront, de ce fait même, entendues; du moins bénéficierai-je d'emblée d'un préjugé favorable et l'on me prêtera une oreille attentive.

\section{L'union sociale}

Commençons par une remarque ayant trait à la référence principale de Taylor, à savoir Humboldt. Il est extrêmement curieux que le philosophe canadien se l'approprie ainsi et en fasse un repoussoir du libéralisme, alors que celui-ci a toujours été, pour autant que nous le sachions, une source typiquement libérale, à laquelle se sont abreuvés Mill, et, pour ce qui nous intéresse plus particulièrement ici, Rawls lui-même ${ }^{4}$. De fait, s'agissant d'expliciter l'idée d'une

4. Dans De la liberté, Mill s'appuie, tout en le réévaluant, sur «le traité de l'éminent savant et politicien Wilhem von Humboldt ", De la sphère et des devoirs du Gouvernement, pour montrer en quoi le développement plein et entier de l'individualité est la fin humaine suprême. Rien d'étonnant à cela dans la mesure où la doctrine du philosophe allemand "attache tant de prix à l'individualité », p. 148. 
société bien ordonnée comme "union sociale d'union sociales ", ce dernier commence par rappeler la paternité de cette notion, qu'il reprend totalement à son compte: "Cette idée vient de von Humboldt ", écrit-il, citant l'Essai pour limiter l'action de l'État, dans lequel le penseur allemand explique que c'est " grâce à l'union sociale, fondée sur les besoins et les capacités internes de ses membres, que chacun peut participer aux richesses collectives de tous les autres $»^{5}$.

Au-delà de la simple référence à Humboldt, ce qu'il est primordial de noter ici est la teneur du propos rawlsien :

Ma thèse est qu'une société bien ordonnée par les deux principes de justice peut représenter, pour chaque citoyen, un bien beaucoup plus global que le bien d'individus laissés à eux-mêmes ou limités à de petites associations. La participation à ce bien global peut élargir considérablement le bien particulier de chaque personne et le renforcer. Le bien représenté par l'union sociale est le plus complètement réalisé quand tout le monde participe [...] (LP, p. 379).

Ainsi, parce que nous sommes des êtres qui ne peuvent se suffire à euxmêmes et vivre en autarcie, nous nous devons de réaliser une union sociale au sein de laquelle complémentarité et coopération seront les maîtres-mots. Mais il y a plus important. La société bien ordonnée régie par les deux principes de justice rawlsiens n'est pas une simple union sociale mais une « union sociale d'unions sociales » (ibid., italique ajouté), comme si le plus petit mode d'être pour l'individu était déjà social, et que seules la taille et la nature de l'association différaient. Comme l'explique Rawls, «ce qui est nécessaire, c'est que chacun puisse faire partie d'au moins une communauté partageant ses intérêts» (TJ, 1987, p. 482 ; italique ajouté $)^{6}$. Ainsi, chacun d'entre nous est membre de diverses unions sociales où il se réalise partiellement à chaque fois en développant ses potentialités et en exerçant ses dons: nous pouvons ainsi être à la fois un joueur de volley-ball doué, un cordon bleu, un ami fidèle et un pédagogue averti, chacune de ces activités constituant une union sociale interactive à laquelle ceux qui excellent (plus ou moins) prennent part. Toutes ces unions sociales plus ou moins élaborées, plus ou moins importantes, dépendent d'une union sociale bien plus étendue qui les contient toutes et qui se trouve être leur condition de possibilité : il s'agit de la société bien ordonnée.

Cette insistance sur la nature sociale de l'humanité n'a rien d'anodin. Elle n'est pas non plus une concession faite à la critique communautarienne. Développée dès $1963^{7}$, elle se trouve au fondement même de la conception

5. LP, p. 379. La référence à Humboldt est tirée des Gesammelte Schriften (Berlin, 1903), livre I, p. 107 sq., comme le mentionne Rawls, ibid. n. 1.

6. Un peu plus loin, Rawls parle même de «communauté de communautés ", TJ, p. 572.

7. Cette psychologie morale figure en effet en bonne place dans "The Sense of Justice " [SJ], article de Rawls datant de 1963. La seule différence notable entre les deux concerne les références: en 1963, le philosophe américain s'inspire de Jean Piaget, tandis que c'est sur la théorie de Kohlberg qu'il se fonde en 1971. 
rawlsienne de la justice, ce que résume parfaitement le lien opéré par le philosophe américain entre justice et coopération sociale:

Dans une société parfaitement juste, les personnes recherchent leur bien d'une façon qui leur est particulière, et donc dépendent de leurs partenaires pour faire ce qu'elles ne pourraient faire ou bien ce qu'elles auraient pu faire mais n'ont pas fait (TJ, p. 572).

Cette interdépendance entre les membres de l'union sociale qu'est la société bien ordonnée rawlsienne implique leur complémentarité, précisément celle dont Taylor louait la présence au sein du modèle associatif humboldtien. De fait, pour Rawls, dans la mesure où tout individu est porteur d'un projet de vie rationnel particulier (lui-même issu d'une conception du bien déterminée) et que l'achèvement de celui-ci dépend de la mise en œuvre de moyens auxquels son détenteur ne saurait à lui seul pourvoir, la complémentarité de l'ensemble des schémas existentiels est la condition sine qua non de la réalisation personnelle. Le propos rawlsien puise indéniablement à la source humboldtienne, et l'on y retrouve de manière prégnante l'idée selon laquelle nul ne peut, en solitaire, réaliser l'humanité, celle-ci étant le fruit du concours de l'ensemble des volontés individuelles ${ }^{8}$.

Rawls spécifie que, si nous devions, par ailleurs, avoir le choix entre deux ou plusieurs projets rationnels d'égale valeur, ceux d'entre eux « qui réalisent les buts d'autrui en même temps que les autres ser[aie]nt préférables » (ibid., p. 466) : le bénéfice de la communauté doit être un critère de premier plan dans la détermination des orientations existentielles des uns et des autres, d'autant que l'estime de soi dépend de l'image valorisante que nous renvoient nos semblables. En effet, "sans l'appréciation de nos concitoyens, il nous est impossible de rester convaincus que nos entreprises ont de la valeur» (ibid., p. 480). L' "approbation de soi ", ainsi que la nommait Adam Smith, ou le " respect de soi », le bien premier le plus important dans la terminologie rawlsienne, ne s'établit sur des bases solides et durables que lorsque les autres reconnaissent que la nature de mes choix et leur hiérarchisation en sous-projets méritent considération et égards. Il paraît donc pour le moins erroné d'opposer l'individu rawlsien à la communauté et de faire de Rawls le chantre d'un " atomisme social» ou d'un monisme réducteurs. Autrui apparaît comme celui sans le regard de qui je ne peux me construire.

\section{Quelle mobilité structurelle?}

Plus fondamentalement, il ne sert à rien de reprocher au libéralisme procédural d'opposer la sphère de l'individu à celle de la communauté parce que nous évoluons structurellement dans une sphère intermédiaire, une sphère proprement

8. Cf. un peu plus loin: «C'est un trait de la sociabilité humaine que nous ne pouvons réaliser par nous mêmes qu'une partie de ce que nous pourrions être. Nous devons compter sur les autres pour réaliser les excellences que nous devons nous-mêmes laisser de côté ou qui nous font défaut ", ibid. 
sociale au sein de laquelle des sympathies se forment, des liens se nouent et d'autres se défont dans une mouvement continu et créateur. Tel est le phénomène d'acclusion selon lequel nous serions dans une mobilité permanente, plus ici mais pas encore là.

Cette vision du social en termes d'espace-flux dans lequel tout se perd, tout se crée et tout se transforme, informe la théorie de la justice comme équité, qui se retrouve plus particulièrement de manière prégnante chez Rawls lorsqu'il est question de la typologie culturelle à l'œuvre dans une société bien ordonnée. Y transparaît l'idée selon laquelle le libéralisme procédural ne procède pas par séparations tranchées et artificielles, mais se fonde sur une porosité et une interpénétration des différentes sphères. On peut ainsi y dénombrer trois types de culture : la culture politique, la culture environnante (ou culture du contexte social) et la culture politique non publique. La première « inclut les valeurs politiques, civiles, autour desquelles se forme le consensus par recoupement, et qui forment la tradition et le fonds d'idées politiques communes ». La "culture politique non publique ", elle, "comprend les médias [...] de tous types: les journaux, les revues et magazines, la télévision et la radio, et beaucoup d'autres" (IRPR, p. 162, n. 13). La culture environnante comprend toutes les doctrines métaphysiques issues du pluralisme raisonnable qui «en constituent la culture sociale et non politique, la culture de la vie quotidienne, de ses nombreuses associations : Églises et Université, sociétés savantes et scientifiques, clubs et équipes, pour n'en nommer que quelques-unes » (LP, p. 38). De ce fait, il ne saurait y avoir de réelle coupure, socialement parlant, entre le métaphysique - que le libéralisme procédural est censé reléguer dans la sphère privée -, et le politique, qui seul aurait droit de cité dans l'espace public. Il existe bel et bien une culture intermédiaire au sein de laquelle se retrouvent les uns et les autres, qui fait le lien entre le politique épuré de toute considération compréhensive et le métaphysique relatif et changeant: «la culture politique non publique établit une médiation entre la culture politique publique et la culture du contexte social » (IRPR, ibid.).

Une difficulté demeure toutefois. Au vu de la circulation, du passage et du changement essentiels qui caractérisent l'existence individuelle se pose la question de la valeur même des différentes unions sociales qui fleurissent au sein de la société bien ordonnée et, partant, celle de la plus grande de toutes, qui les englobe et les conditionne, à savoir l'État. En ce sens, le problème n'est pas tant cette tendance structurelle à la mobilité que la manière d'en envisager les modalités. De fait, c'est une chose que de faire le diagnostic de la mobilité continue, c'en est une autre de la justifier et d'en faire ressortir la positivité quand pour ce faire on invoque le droit de l'individu à choisir ses appartenances ${ }^{9}$. De fait, si les communautariens sont rarement perméables à

9. Plus que cela, revient souvent chez Walzer l'idée que la mobilité reste dans la plupart des cas contrainte et forcée : «Les êtres humains, comme je l'ai dit déjà dit, se déplacent beaucoup, mais pas parce qu'ils aiment se déplacer. Ils sont, pour la plupart, enclins à rester là où ils sont à moins que leur vie ne soit très difficile dans l'endroit en question » (1997c, p. 70). 
cette sensibilité altruiste développée par le libéralisme procédural que l'on a tenté de mettre au jour jusqu'ici, c'est parce qu'elle n'est au fond que l'arbre qui cache la forêt, une forêt de tous les dangers. Ce qu'ils dénoncent est bien plus grave que le simple égoïsme dont ferait preuve l'individu libéral: c'est en effet l'idée de priorité du moi sur ses fins qui concentre les critiques communautariennes les plus virulentes. Celles-ci y voient une aberration conceptuelle doublée d'un extraordinaire irréalisme: comment l'individu pourrait-il être à ce point «désencombré » que tous ses choix et tous ses engagements dépendraient exclusivement de sa propre capacité de choix ?

C'est précisément là le cheval de bataille enfourché par Walzer, à qui l'on doit la formulation la plus pertinente de l'embarras communautarien devant la mobilité des appartenances de l'individu libéral que rien ne retient nulle part. Ainsi, après une étude sociologique qui établit que l'Américain est soumis à quatre types de mobilités (politique, sociale, géographique et matrimoniale), Walzer relativise ce changement et ce mouvement en montrant, par exemple, que « de nos jours encore, dans cette société si libérale et si mobile, il est toujours vrai que la meilleure façon de prévoir le vote des gens est de savoir comment votaient leurs parents ». Il en vient dès lors, et dans un premier temps, à rechercher des «indices de communauté » (Walzer, 1997a, p. 70) dans la philosophie libérale, stigmatisant ceux qui dénoncent l'irréalisme de celle-ci. Il affine ensuite son argument, montrant que ce qui dérange est le fait, non que les libéraux appellent de leurs vœux la dissociation, la séparation, la coupure ou le changement, mais bien plutôt qu'ils considèrent l'appartenance à des groupes comme purement volontaire, ce qui explique qu'ils puissent $a$ priori se défaire de tout lien: "c'est une erreur (très caractéristique du libéralisme) de penser que les formes d'association existantes soient entièrement, voire largement, volontaires et contractuelles, c'est-à-dire le résultat de la seule volonté » (ibid.). En effet, les groupes et les appartenances que nous semblons choisir parmi un panel de liens existants «ne sont que l'expression de ces identités sous-jacentes qui, encore une fois, ne sont pas tant choisies que mises en œuvre»(ibid.).

Tout naturellement, le philosophe communautarien en vient à l'examen de l'union sociale des unions sociales conçue par Rawls. Celle-ci représenterait, selon lui, la conception (voire la concession) la plus aboutie de l'identité à laquelle le libéralisme peut prétendre. Mais l'union sociale ainsi envisagée porte nécessairement en elle ses propres limites. Le libéralisme aurait alors le choix entre se résigner à un État en manque de stabilité, miné par les allers-retours de ses membres d'un groupe à un autre, ou outrepasser ses prérogatives pour tenter de renforcer la cohésion en son sein, en reniant son exigence de neutralité vis-à-vis des conceptions de la vie bonne, de sorte qu'il finirait par tendre vers une forme de perfectionnisme - qu'au demeurant Walzer considère comme ce qui pourrait arriver de mieux à un État libéral (ou socialdémocrate) digne de ce nom ${ }^{10}$ :

10. Ibid., p. 73. 
Mais si tout groupe est précaire, à deux doigts de la dissolution ou de l'abandon, alors certainement, l'union plus large doit également être faible et vulnérable. Ou alors, alternativement, ses chefs et représentants vont être poussés à compenser ailleurs les défauts de l'association en renforçant leur propre union, c'està-dire l'État central, au-delà des limites permises par le libéralisme (ibid., p. 71).

Confrontons le présupposé de Walzer, selon lequel le libéralisme considère nos appartenances et les liens que nous choisissons comme le fruit d'une décision volontaire et délibérée, à la définition que donne Rawls de sa société bien ordonnée ${ }^{11}$. Selon l'auteur de Libéralisme politique, cette dernière n'est pas une association: " une société bien ordonnée n'est ni une communauté ni, plus généralement, une association » $(L P, \text { p. 67 })^{12}$. Nous ne choisissons pas d'y entrer ou d'en sortir, comme c'est le cas pour tout regroupement volontaire, mais « on y entre seulement à la naissance, et on en sort seulement à la mort » $(i b i d .)^{13}$. Il est en ce sens primordial de distinguer entre la position originelle comme procédure heuristique, «artifice de représentation » purement monologique ou expérience de pensée dans laquelle nous entrons (ou non) volontairement, de la réalité de l'existence humaine. C'est bien parce qu'il différencie la première de la seconde que Rawls est en mesure de déclarer que «nous

11. Nous n'examinerons pas ici l'idée de Walzer selon laquelle les unions sociales qui existent dans la société libérale sont par définition fragiles, portant en elles fatalement une propension à la dissolution qui viendra à l'être un jour ou l'autre (et visiblement plutôt aujourd'hui que demain). Cela apparaît nettement lorsque le philosophe affirme que «la meilleure façon de comprendre le libéralisme est de le voir comme une théorie des liens entre personnes, qui a pour centre l'association volontaire et qui interprète 'volontaire' comme étant le droit de rompre ou de se retirer (ibid., p. 81). Ainsi, explique-t-il, ce qui rend un mariage volontaire, c'est la possibilité permanente de divorcer" (ibid.). On peut aisément débusquer le postulat qui sous-tend ce type d'affirmation: dans la mesure où c'est l'intérêt qui guide les choix de l'individu libéral et que ses appartenances ne sont exclusivement décidées qu'après force calculs et computations, pareil personnage vogue de groupe en groupe au gré du profit qu'il est en mesure d'en tirer, unique critère auquel répondent ses affiliations. De là, comme de juste, délitements, décompositions et désagrégations à foison mais, également, des comportements resquilleurs consistant à profiter du système sans contrepartie " ces gens qui continuent de profiter des avantages de l'adhésion et de l'identité tout en ne participant plus aux activités qui produisent ces avantages " (Walzer, ibid., p. 71). Tout cela sera examiné dans les développements qui suivent, lorsqu'il sera question de l'identité collective et du problème de savoir si l'anthropologie libérale est à même d'en fonder une quelconque conception.

12. Rawls définit la communauté comme « une forme particulière d'association unifiée par une doctrine compréhensive» $(L P$, p. 67, n. 1$)$.

13. Rawls met en avant une seconde différence entre la société bien ordonnée telle qu'il la conçoit, et une association : cette dernière peut tout à fait être inéquitable et favoriser certains aux détriments des autres. De ce fait, la société bien ordonnée «n’a pas le droit, comme le font en général les associations dans le cadre de la société, d'offrir des conditions différentes à ces membres (dans ce cas, ceux qui sont nés dans la société) en fonction de la valeur de leur contribution potentielle à la société dans son ensemble, ou aux fins de ceux qui en sont déjà membres $(L P$, p. 69). 
n'avons pas d'identité antérieure à notre existence sociale » $(\text { ibid. })^{14}$. En ce sens, " la seconde critique communautarienne ", celle qui "soutient que la théorie libérale mésinterprète la vie réelle » en affirmant que les hommes sont foncièrement des êtres désengagés et monades repliés sur eux-mêmes, se trompe au moins sur ce point (Walzer, ibid., p. 59) ${ }^{15}$. Notre identité est avant tout sociale, et le lien qui nous attache à l'union sociale des unions sociales n'est pas un lien contractuel parce que la société bien ordonnée n'est pas une association. De ce fait, contrairement à ce qu'affirme Walzer, l'union sociale englobante, condition de possibilité de toutes les autres, ne saurait être de même type que celles qu'elle abrite en son sein ; la différence de taille s'accompagne d'une différence autrement plus importante : une différence de nature. Dès lors, le lien de cause à effet mis en avant par le philosophe communautarien, lien selon lequel de la fragilité des unions sociales découle la vulnérabilité de l'union sociale par excellence qu'est l'État, semble sérieusement mis à mal. Au demeurant, et indépendamment de cela, l'on voit mal comment actualiser son talent pour le basket-ball à un moment donné de son existence et faire le choix de mettre fin à la pratique de ce sport ultérieurement (ou, si l'exemple choisi paraît trop trivial, récuser la religion de ses parents que l'on a reçu en héritage et décider d'évoluer vers un athéisme, un « simple » déisme ou une autre religion), aurait des conséquences négatives sur la stabilité de l’État. La seule manière de comprendre le lien que fait Walzer entre les deux est d'avancer que la facilité avec laquelle je me défais de mes appartenances et de mes liens, conçue comme structure mentale, se retrouve dans le choix que je pourrais faire de

14. Nous avions déjà opposé à Taylor une autre formulation de cette même idée selon laquelle «le plus petit mode d'être de l'individu est déjà social ", voir supra.

15. Walzer ne partage pas cette critique; il souligne qu'il serait aberrant d'affirmer que «les libéraux d'aujourd'hui [...] croient [...] en un soi présocial (1997a, p. 80). Précisons que le philosophe distingue en effet entre deux critiques communautariennes (en montrant les limites de chacune): la première considère qu'il y a une adéquation entre la réalité que vivent les hommes aujourd'hui (séparés les uns des autres et libres de tout engagement), c.-à-d. la «pratique libérale « (ibid., p. 57), et la théorie politique libérale défendant l'idée que le moi préexiste à ses fins ; cette critique, comme de juste, récuse l'ensemble formé par l'une et l'autre. La seconde critique insiste au contraire sur l'idée que nous sommes tous mus par des liens forts, des attaches substantielles qui font notre quotidien et stigmatise le fait que le libéralisme, imperméable à tout bon sens, est aveugle à cette réalité, persistant à affirmer la primauté du moi sur ses fins et à concevoir les rapports qui nous lient les uns aux autres comme purement conjoncturels. 
quitter l'union sociale des unions sociales. Or, comme nous venons de le voir, cela est totalement rejeté par Rawls ${ }^{16}$.

\section{Quelle identité collective?}

Cela étant, un problème de taille subsiste : dans cette "association » de type humboldtien, de quelle nature serait «l'entreprise commune », pour employer la terminologie taylorienne, qui mobiliserait tous les membres du groupe ${ }^{17}$ ? Cette interrogation soulève un épineux problème, celui de l'identité collective. De fait, pour regarder ensemble dans une même direction, il faut que nous soyons mus par un sentiment puissant, celui d'une appartenance commune, source d'un destin commun ${ }^{18}$. Or, dans une conception purement politique de la justice, autour de quel idéal fédérateur et constitutif les membres de la société bien ordonnée de Rawls peuvent-ils se retrouver? Le libéralisme procédural peut-il accoucher d'une conception forte de l'identité collective sans trahir son credo, à savoir l'incapacité foncière du Bien, quel qu'il soit, à fonder des normes politiques authentiquement justes? Y a-t-il, pour reprendre la question de Taylor, un «patriotisme libéral » ? C'est la pertinence de cette alliance a priori improbable qui va à présent retenir notre attention.

16. Ailleurs, toutefois, dans une analyse extrêmement judicieuse de l'identité personnelle à l'heure d'une postmodernité portant encore en elle les germes de la modernité, Walzer reconnaît que ce qui caractérise l'individu qui vit au sein d'une société démocratique libérale est d'être (aussi) un "dissident ", un "ambivalent " (Walzer, 1998, p. 133), un "vagabond culturel» (ibid., p. 160), «citoyen d'un État et membre d'un groupe, mais aussi [...] étranger à l'un comme à l'autre» (ibid., p. 132). Les analyses et descriptions sociologiques, historiques et anthropologiques de Walzer sont toujours fort justes, mais transparait immanquablement sous une forme plus ou moins explicite l'idée que des ajustements sont nécessaires, que seul le communautarisme (que par ailleurs il n'hésite pas à renvoyer dos à dos avec le libéralisme) est à même d'opérer. Aussi affirme-t-il de manière réitérée que des "correctifs communautariens " sont nécessaires au libéralisme (Walzer, 1997a, p. 81) ; pour l'exemple précis cité ci-dessus, le délitement et la dispersion sont considérés comme des conséquences néfastes du libéralisme qu'il faut veiller à endiguer en insufflant du lien là où tout menace de se dissoudre ou se distendre, un lien entre modernité et post-modernité, entre identité civique et identité(s) «privée(s) " (Walzer, 1998, p. 134). Pour autant, l'étude bienveillante d'un certain libéralisme peut nous dispenser de ce recours au communautarisme. Ce à quoi Walzer, qui décidément n'a pas peur de la contradiction, souscrit totalement. De fait, avance-t-il, si le modèle libéral a créé, comme le stigmatise la critique communautarienne, une société fortement décomposée et "désencombrée ", c'est à lui, peut-on raisonnablement penser, que reviennent à la fois la responsabilité et la légitimité de sa remise en cause et de son éventuel amendement: «[...] nous pouvons supposer que la politique libérale est la mieux armée pour faire face aux conséquences problématiques de cette décomposition» (Walzer, 1997a, p. 58).

17. Nous mettons le terme "association" entre guillemets, car Rawls récuse l'idée que la société bien ordonnée régie par ses deux principes de justice soit une association au sens d'un groupe dans lequel on entre volontairement. Je l'utilise ici dans son sens commun de rassemblement.

18. Tout pays démocratique a besoin d'une identité commune au sens de "forme » dans laquelle les citoyens se reconnaissent comme appartenant ensemble à un même groupe. Ils se reconnaissent comme liés avec leurs concitoyens dans un même projet [...] qui les lie en une unité, en un agent commun» (Taylor, 1999, p. 59). 
S'interrogeant précisément sur la nature de « la forme » à même de fédérer tout un peuple, le philosophe canadien constate que l'ère du " patriotisme constitutionnel » semble être révolue depuis que des pays comme la France et les États-Unis, pourtant traditionnellement unifiés autour de principes exclusivement politiques, évoluent vers une compréhension d'eux-mêmes en termes culturels et ethniques, ressentant «de plus en plus le besoin de réagir à leurs propres symboles pour sentir leur unité » (ibid., p. 61). Cela signifie que, quand bien même le libéralisme procédural aurait eu une conception valide de l'identité collective (c.-à-d., penser, créer et défendre des règles justes et légitimes), celle-ci se révèle aujourd'hui obsolète parce qu'incapable de fédérer la diversité des sociétés post-modernes. La faillite du «patriotisme constitutionnel » en tant que modèle identitaire viable démontre ainsi que les peuples prennent conscience, idée éminemment moderne pour le coup, que leur identité existe indépendamment de la structure politique qui les informe à un moment donné de leur histoire: « on trouve le principe d'unité ailleurs que dans le simple fait de la positivité politique » (ibid., p. 62-63). De là, comme de juste, la quête de la reconnaissance qui anime les minorités endogènes ou exogènes à l'intérieur d'une même nation : si la modernité s'est battue pour l'égalité, il faut à présent qu'elle assume les conséquences de son statut de valeur des valeurs.

Pourtant, dans un article daté de 1997, Taylor, s'intéressant à la notion de patriotisme, tentait de comprendre ce que pouvait signifier, pour le libéralisme procédural, qu'un individu agisse en patriote. De manière fort judicieuse, le philosophe canadien montre qu'il serait totalement abusif de dénier l'existence d'un "régime patriotique libéral » sous prétexte que "le libéralisme procédural impliqu[er]ait une ontologie atomiste parce qu'il parle des plans de vie individuels » (p. 110). Il existe bel et bien « un patriotisme du droit », authentiquement libéral (ibid.). Réfléchissant sur la teneur de celui-ci, c'est-à-dire, au fond, aux limites dans lesquelles il doit impérativement se cantonner pour espérer rester fidèle aux réquisits et aux valeurs maîtresses du libéralisme procédural, Taylor affirme que ce dernier se démarque du républicanisme et plus précisément de sa version forte, l'humanisme civique, par son rejet du gouvernement des citoyens par eux-mêmes comme fin en soi. De fait, considérer ce dernier comme le bien plus important de la vie humaine, reviendrait, pour le libéralisme procédural, à avaliser une conception particulière de la vie bonne, ce qui contredirait totalement l'exigence de neutralité sur laquelle il se fonde :

Ce qui est laissé de côté ici [par le libéralisme procédural], c'est [...] l'autogouvernement participatif [...] Les libéraux procéduraux tendent à le négliger, [le] traitant $[. .$.$] comme purement instrumental à l'égard de l'État de droit et de$ l'égalité. En effet, traiter l'autogouvernement [...] comme le bien le plus précieux en soi nous amènerait au-delà des limites du libéralisme procédural, parce qu'une société organisée autour de cette proposition partagerait et sanctionnerait au moins cette proposition à propos de la vie bonne (Taylor, 1997, p. 112-113).

En effet, dans l'esprit du modèle libéralo-procédural, « les institutions ont une signification entièrement instrumentale ", de sorte que la participation n'a 
aucune valeur (ibid., 114). Cela s'explique par le fait que, lorsqu'il y a possibilité de bénéficier d'un système de contrepoids face aux instances gouvernementales (ce qui, dans une démocratie libérale, est tout de même plus souvent le cas que l'inverse), le désengagement civique n'a pas d'incidence sur la liberté des individus. En d'autres termes, si l'on a les moyens de se prémunir des ratés ou des dangers du système, si l'on possède une certaine "influence ", l'idéal d'autogouvernement apparaît comme totalement superflu (ibid.)

Taylor s'interroge sur la viabilité et la valeur des deux modèles patriotiques ainsi définis. Très justement, il montre que celles-ci, dans le fond, dépendent des "penchants" (ibid., p. 115) des uns et des autres, et que l'heure est à la surenchère argumentative, plus qu'à une résolution définitive de l'alternative en faveur de l'une ou l'autre tradition. C'est là une conclusion pleine de bon sens. Toutefois, il semble, contre Taylor, qu'une « entreprise commune ", authentiquement non formelle, dans laquelle les institutions ne sont pas exclusivement instrumentales - c'est-à-dire conçues uniquement comme de simples moyens en vue de la réalisation des intérêts privés - , puisse tout à fait exister dans une version bien déterminée du libéralisme procédural. Ainsi en est-il de la vision de la société bien ordonnée rawlsienne; à ce titre, la question de l'identité collective ne saurait être étrangère au libéralisme (politique) prôné par le philosophe américain.

Dans L'idée de raison publique reconsidérée, le lecteur est frappé par la tonalité du propos de Rawls lorsque ce dernier s'attache à expliciter la manière dont les citoyens ordinaires - entendons par là ceux qui ne sont pas investis d'une charge politique - doivent réaliser l'idéal de la raison publique :

[...] nous proposons que les citoyens doivent idéalement se concevoir comme s'ils étaient des législateurs et se demander quelles lois, justifiées par quelles raisons conformes au critère de réciprocité, ils estimeraient tout à fait raisonnable d'adopter (IRPR, p. 163 ; l'italique est de Rawls).

Ce qu'il est important de noter ici, c'est l'idée que tout un chacun doit se sentir investi d'une mission législatrice, au même titre que n'importe quel responsable politique, c'est-à-dire avec le même sens de la responsabilité, avec le sentiment aigu que se joue, à chaque fois, quelque chose de fondamental ${ }^{19}$. De surcroît, le devoir de justice est bien un "devoir moral intrinsèque " (ibid., p. 164) commandant une vigilance de tous les instants pour que l'idéal de raison publique ne soit pas malmené par des responsables politiques peu scrupuleux : les citoyens doivent veiller à « se défaire » de ceux qui ne font pas honneur à la charge politique qu'ils exercent ou au poste public qu'ils briguent, et faire ainsi « leur possible pour obliger [les responsables gouvernementaux] à [soutenir l'idée de raison publique] " (ibid.) Nous sommes bien loin, ce faisant, d'une instrumentalisation des institutions au profit des 
intérêts privés et relativement proches, a contrario, de l'idée d'autogouvernement participatif que Taylor attribuait exclusivement à l'humanisme civique, ainsi que de la hantise authentiquement républicaine du spectre de la corruption ${ }^{20}$.

Cette idée gagne en profondeur lorsque l'on s'intéresse plus précisément aux spécifications rawlsiennes du devoir de justice. Celui-ci est défini comme un "devoir naturel », commandant de "protéger et respecter les institutions justes qui existent et s'appliquent à tous » (TJ, p. 145). Mais les développements qui suivent ont de quoi étonner. Ce devoir de justice, peut-on lire, «nous contraint aussi de promouvoir des organisations justes qui pourtant n'existent pas encore, du moins si c'est possible à un coût qui n'est pas trop élevé pour nous-mêmes " (ibid.; l'italique est de nous). Cela signifie que la citoyenneté telle qu'elle est envisagée au sein de la théorie de la justice comme équité n'est pas un simple statut; elle n'est pas cette pure passivité censée être caractéristique du libéralisme, et que les tenants du républicanisme condamnent, appelant de leurs vœux des temps révolus où la vertu civique n'était pas un vain mot. Être un citoyen, c'est être (aussi) un créateur; l'humanisme civique néo-aristotélicien ne disait pas autre chose ${ }^{21}$.

Comment comprendre toutefois la restriction apportée par Rawls, selon laquelle le devoir de justice ne nous invite pas à faire preuve d'un zèle civique à tout prix ? D'aucuns verraient sans doute que la condition finale énoncée ici tempère sérieusement l'idée selon laquelle la société bien ordonnée promeut l'engagement civique et lui confère une place centrale dans la mesure où l'investissement individuel dépendrait toujours d'un calcul des risques éventuels et d'une estimation du prix à payer. Il serait plus juste, semble t-il, de voir dans ce bémol un rejet typiquement libéral du surérogatoire plutôt que la volonté délibérée d'instrumentaliser l'engagement civique.

Mais ce qu'il est intéressant de remarquer est le fait que Rawls, dans la préface à l'édition paperback de Political Liberalism, ne récuse plus aussi radicalement une participation civique surérogatoire se faisant au détriment de l'intérêt personnel. Il affirme que les citoyens sont d'accord pour agir conformément aux termes de la coopération sociale sur les bases de laquelle ils se sont entendus au préalable et qu'ils ont tous entérinée et ce, "même au prix de leurs propres intérêts dans certaines situations " (PL, p. xlii. L'italique est de nous, ainsi que la traduction $)^{22}$. Le caractère sacré de l'engagement civique qui a permis de bâtir et de promouvoir des institutions solides est, en dernière instance, incommensurable et explique qu'un «citoyen, qui vote selon ses

20. Voir à ce sujet Skinner (1984).

21. Nous ne disons pas que Rawls est un humaniste civique. Il s'en défend d'ailleurs explicitement dans $L P$, affirmant sa sympathie pour le républicanisme classique d'inspiration néoromaine, mais rejetant sa version extrémiste, néo-aristotélicienne. Nous disons bien plutôt que sa théorie est traversée de part en part de moments où est affirmée une conception forte de la citoyenneté à laquelle on trouve des échos dans la tradition de l'humanisme civique.

22. Cette introduction n'est pas reproduite dans la traduction française de $L P$. 
principes moraux (sa conception de la justice), accepte la décision de la majorité lorsqu'il se trouve lui-même dans la minorité »(LODFP, p. 119). De fait, «il doit voter pour ce qu'il considère être la meilleure mesure, sans s'occuper du résultat du vote ", car la procédure constitutionnelle "ne produit pas une affirmation destinée à être crue [...] mais une règle qui doit être suivie » (ibid., p. $120 ; 122$ ). Telles sont les exigences du devoir de fair-play, que Rawls explicite par une hypothèse :

Supposons qu'il existe un système de coopération mutuellement bénéfique et juste et que le profit dont il est porteur ne puisse être réalisé que par la coopération de tous, ou presque. Supposons, de plus, que cette coopération exige un certain sacrifice de la part de chaque personne ou, à tout le moins, engage une certaine restriction de sa liberté. Supposons, enfin, que les bénéfices produits par la coopération soient, jusqu'à un certain point, gratuits : à savoir que le système de coopération est instable dans le sens où, si une personne venait à savoir que toutes les autres (ou presque) continuaient à apporter leur contribution, elle serait encore en mesure de tirer profit du système même si elle s'abstenait d'apporter la sienne. Sous ces conditions, une personne qui a accepté les avantages du système est tenue par un devoir de fair-play d'y contribuer et de ne pas tirer avantage du profit gratuit en ne coopérant pas. La raison pour laquelle l'on doit s'abstenir [de ce type de comportement] est que l'existence du bénéfice résulte de l'effort de chacun et qu'avant de [chercher à] comprendre comment celui-ci doit être partagé, si d'aventure il devait l'être, [l'on doit avoir à l'esprit qu'il] ne profite, en toute équité, à personne (ibid.; notre traduction).

Voilà, à tout le moins, une belle démonstration du respect de l'institution qui montre que libéralisme procédural ne rime pas avec recherche de l'intérêt privé à tout prix, mépris et instrumentalisation des autres et du système. Il y a là beaucoup plus que le simple souci de se prémunir du free-rider ou resquilleur, figure que le libéralisme a toujours cherché à maintenir à distance. En effet, pour qui s'intéresse à la psychologie morale développée par Rawls, il apparaît rapidement que la manipulation et la tromperie, deux caractéristiques fondamentales de la resquille, ne sauraient faire partie de la structure mentale des citoyens d'une société bien ordonnée, indépendamment du fondement théorique de celle-ci, libéral ou non libéral. Cela ne s'explique pas par une évacuation pure et simple des sentiments négatifs qui gêneraient l'application d'une justice idéale, fondée sur l'angélisme irréaliste des divers acteurs sociaux, mais bien plutôt par le fait que les individus passent par différents stades moraux qui les façonnent de sorte que l'envie de profiter des autres disparaît progressivement.

Ainsi, après avoir traversé la première étape du développement moral, dans laquelle le caprice infantile se confronte à l'autorité légitime des parents, les enfants passent par le deuxième stade moral, à savoir la morale de groupe, qui les modèle pour en faire des adultes responsables. "Le contenu de la morale de groupe est fourni par les normes morales qui correspondent au rôle de l'individu dans les divers groupes auxquels il appartient » (TJ, p. 508). On retrouve 
ici l'idée que les personnes appartiennent simultanément à plusieurs unions sociales, et que des « liens [d'] amitié et de confiance mutuelle » naissent du commerce avec les autres au sein de ces groupes, et des valeurs d'échange et de partage qu'ils véhiculent (ibid., p. 510). Constater que les uns et les autres « respectent leurs devoirs et leurs obligations d'une manière intentionnelle » (ibid.) fait naître en chacun des membres d'un groupe donné une affection et une loyauté indéfectibles, de sorte que si quelqu'un faillit à son devoir à l'intérieur de l'union sociale considérée, il se sentira envahi par un sentiment de culpabilité, précisément parce qu'il aura trabi de tels sentiments et non pas parce qu'il se sera fait prendre. Or un resquilleur, un manipulateur ou un parasite n'éprouvent ni culpabilité ni remords pour la simple raison qu'ils ne partagent rien avec les autres et qu'ils ne se sont de ce fait créé, au sein du groupe, ni affinités, ni sympathies, ni attaches.

Cette morale de groupe, continue Rawls, forme structurellement la personnalité des individus, de sorte que ces derniers finissent par acquérir une certaine notion du juste. Ils passent alors à l'ultime stade de développement moral, celui de la «morale fondée sur des principes » (ibid., p. 513). Celui qui aspirait à être un bon époux, un bon joueur de base-ball ou un père accompli veut à présent être «une personne juste» (ibid., p. 513). Nous continuons à être liés par des liens d'amitié et de confiance forts, mais se crée petit à petit une propension à obéir aux normes du juste indépendantes de ces attaches contingentes et particulières (ibid., p. 515). C'est ainsi qu'il faut comprendre le devoir de justice évoqué plus haut : les citoyens de la société bien ordonnée rawlsienne partagent un même souci autour duquel ils se retrouvent et se reconnaissent : créer et protéger des institutions justes, œuvrant par-là même "au développement du sens de la justice pour lui-même ", et non pour des intérêts purement privés, comme le ferait un manipulateur ou un profiteur (LP, p. 379; l'italique est de nous). La citoyenneté, assurément, n'est pas du tout celle préconisée par Montesquieu, pour qui la liberté politique se confond avec la sécurité ou l'idée que nous jouissons de celle-ci' ${ }^{23}$.

Il est intéressant de noter comment, dans le Droit des peuples, ouvrage de Rawls qui élargit sa conception de la justice locale aux questions de justice internationale, la question de l'identité collective est thématisée d'une manière qui, tout à la fois, rejoint et prolonge sa vision d'une citoyenneté forte. Le philosophe américain commence en effet par récuser le terme d'État au profit de celui de peuple, ce qui n'a rien d'anodin : il s'agit, naturellement, de s'intéresser à quelque chose de plus que la simple structure institutionnelle d'un pays, mais pas seulement ${ }^{24}$. En effet, plus un État, aveuglé par la poursuite de ses intérêts égoïstes, fait primer le rationnel sur le raisonnable dans ses relations

23. Voir L'Esprit des lois, XII, 2.

24. Étant entendu que, même si l'inverse reste vrai, tous les États ne peuvent prétendre à être considérés comme des peuples. 
avec les autres États, méprisant ce faisant le «critère de réciprocité ", plus la différence entre État et peuple se creuse. Il faut entendre par là que le second est doté d'un «caractère moral» incommensurable qui lui interdit toute visée hégémonique, conçue ici comme manifestation de la politique de l'État, une politique purement rationnelle. Ce dernier ne peut donc, on l'aura compris, instrumentaliser le peuple qui vit à l'intérieur de ses frontières en invoquant le concept classique de souveraineté : à part les guerres proprement auto-défensives il ne justifie pas de menacer la sécurité d'un État quel qu'il soit $^{25}$.

Le peuple est en effet mû par ce que Rawls nomme le respect de soi, qu'il apparente à l'amour de soi dans son acception rousseauiste. Défini comme «la conscience commune de ses membres des épreuves traversées au cours de leur histoire ainsi que de leur culture et de ses réalisations ", pareil sentiment se lit "dans l'insistance qu'un peuple met à recevoir un respect approprié et une reconnaissance de son égalité de la part des autres peuples» (ibid., p. 50; italique ajouté). C'est dire si l'image que lui renvoient ces derniers importe à un peuple; il ne s'agit pas simplement de faire preuve de rectitude morale dans les relations qu'il entretient avec eux, mais de veiller sans relâche à en obtenir le respect, qui n'est au final pas autre chose que l'exigence d'un traitement d'égal à égal ${ }^{26}$. Autant dire que toute velléité de grandeur (au sens figuré mais aussi au sens propre) qui se manifesterait aux dépens d'autres peuples, est, pour cette raison même, impensable.

Tout cela explique que Rawls soit naturellement porté vers l'idée de nation et qu'il se reconnaisse tout à fait dans les termes par lesquels Mill, au chapitre XVI des Considérations sur le gouvernement représentatif, qualifie et décrit la culture d'un peuple:

On peut dire qu'une portion de l'humanité constitue une nationalité si ses membres sont unis entre eux par des sympathies communes qui n'existent pas entre eux et les autres - qui les amène à coopérer entre eux plus volontiers qu'avec d'autres, à souhaiter être placés sous l'autorité d'un même gouvernement, et à désirer qu'il s'agisse exclusivement d'un gouvernement par euxmêmes, ou une portion d'eux-mêmes. Ce sentiment de nationalité peut avoir été produit par des facteurs variés. Il est parfois l'effet de l'identité de race ou de descendance. Une communauté de langage ou de religion y contribue largement. Les limites géographiques sont aussi l'une de ses causes. La plus puissante est néanmoins l'identité des antécédents politiques, la possession d'une histoire nationale, et la communauté de souvenirs qui en résulte, la fierté et l'humiliation collective, le plaisir et le regret liés aux mêmes événements du passé.

25. DP, p. 31 et 41.

26. C'est précisément cette idée que développe Taylor (1994) lorsqu'il affirme qu'« une personne ou un groupe de personnes peuvent subir un dommage ou une déformation réelle si les gens ou la société qui les entourent leur renvoient une image limitée, avilissante ou méprisable d'eux-mêmes ", p. 41. 
Aucune de ces circonstances n'est cependant suffisante en elle-même (DP, n. 17, p. 37-38).

Avec ces "sympathies communes ", ces sentiments de "fierté et d'humiliation collective ", de " plaisir » et de " regret » éprouvés s'agissant des épisodes marquants d'un vécu que l'on perçoit comme nous étant commun, il semble que nous soyons, encore une fois, fort loin du patriotisme constitutionnel censé être, d'après Taylor, tout à la fois ce à quoi les régimes libéraux peuvent prétendre de mieux et ce dont ils sont dans l'incapacité même de se prévaloir, eu égard à la mixité ethnico-culturelle au sein de laquelle ils évoluent. De fait, le sentiment patriotique n'est en rien incompatible avec l'existence d'une démocratie constitutionnelle qui, en tant que telle, ne possède pas de doctrine englobante du bien ${ }^{27}$. En outre, et parce qu'il est en partie appelé par la disposition morale du peuple, il est tout à fait naturel : " un peuple peut être fier de son histoire et de ses réalisations, comme le permet un patriotisme légitime » (ibid., p. 63 ; c'est Rawls qui souligne), c'est-à-dire sans faire montre d'un quelconque complexe de supériorité par rapport aux autres peuples ${ }^{28}$. Le peuple de Rawls est donc fondamentalement une nation, caractérisée par un modèle de valeurs culturelles, une histoire et des hauts faits dont il est tout à fait légitime de se targuer tant que l'arrogance, sous toutes ses formes, ne vient pas supplanter la fierté.

\section{Nation, peuple et communauté}

En développant ce point, Rawls reprend à son compte, de son propre aveu, le concept de nationalisme libéral tel que l'expose Yael Tamir ${ }^{29}$. Cette référence apporte un éclairage pour le moins conséquent au problème qui nous intéresse ici et nous aide à comprendre, s'agissant de Rawls en particulier, qu'il ne saurait y avoir aucun paradoxe à promouvoir dans le même temps l'idée d'une communauté nationale et un libéralisme politique - à condition, naturellement, de redéfinir les termes de la question ${ }^{30}$. C'est précisément ce à quoi Tamir s'attelle dans cet ouvrage, tentant de comprendre " de quelle

27. Voir DP, p. 55.

28. Sur ce dernier point, voir DP, p. 64.

29. "En pensant l'idée de nation de cette façon, je suis l'ouvrage très instructif de Yael Tamir, Liberal Nationalism (Princeton University Press, Princeton, 1993) » n. 20, p. 39. Néanmoins, Rawls et Tamir divergent sur un point essentiel : pour Rawls, comme on le verra, se défaire totalement des liens qui nous lient aux autres en tant que membres d'un même peuple, tout comme quitter sa nation sont deux comportements sinon impensables du moins très difficilement réalisables, tandis que pour Tamir, «le nationalisme libéral [...] affirme que l'appartenance à une nation est affaire de choix (elective) dans la mesure où les individus peuvent quitter la nation de leur naissance et créer de nouvelles affiliations [...] ", p. 87.

30. Berlin (1988) montrait déjà — même s'il se place dans une perspective fort différente - l'urgence qu'il y avait à prendre en considération sinon le nationalisme, du moins la «conscience nationale» (p. 361) : "On peut dire, je pense, sans aucune exagération, que nul mouvement politique, de nos jours, n'aurait la moindre chance de succès s'il refusait de s'appuyer sur le sentiment national », p. 372. 
manière le nationalisme peut offrir [son aide] à la pensée libérale » (1993, p. 4). Il s'agit, au fond, de montrer que l'opposition classique entre les deux courants est à la fois «simpliste et trompeuse»(p. 5)

Ainsi, si l'appartenance nationale (et donc culturelle) n'est pas initialement affaire de choix mais de naissance, cela ne saurait impliquer l'absence et le refus de l'autonomie individuelle, de la réflexion, voire de la critique. Mais inversement, postuler ces valeurs typiquement libérales - unies autour du principe de la priorité du moi sur ses fins - ne revient en aucun cas à affirmer que le sentiment national d'appartenir à une communauté unie par une langue, une culture et des souvenirs communs, est "superficiel », voire «totalement non pertinent dans la définition de soi» (p. 7) : l'identité est un processus qui combine découverte et choix ${ }^{32}$. Le choix et le recul sont, peuton alors penser, la condition de la persistance dans le temps de l'identité nationale, éléments sans lesquels une actualisation de celle-ci (au sens aristotélicien et contemporain du terme) serait inenvisageable. Ce qui compte dès lors n'est pas tant la substance (ou le caractère) de la culture, que sa structure, qui, comme l'affirme Dworkin, nous fournit les conditions ou le cadre nécessaires pour prendre des décisions potentielles concernant la manière dont nous devons conduire nos vies; une structure qui, affirme-t-il, peut être enrichie ou appauvrie s'agissant des opportunités qu'elle propose ${ }^{33}$. Ainsi, lorsque le philosophe américain s'exprime sans état d'âme au sujet de la force de l'appartenance culturelle, il faut y voir un réalisme très juste plutôt que la manifestation d'un nationalisme exacerbé, tant s'en faut: "Les liens qui se nouent avec les personnes et les lieux, les associations et les communautés, ainsi que les liens culturels sont en général trop forts pour être abandonnés, et ce fait ne doit pas être déploré » (LP, p. 331).

Comme la majeure partie des théoriciens libéraux, Rawls ne commande pas aux personnes de s'arracher d'elles-mêmes au nom du credo de la priorité du moi sur ses fins et, ce faisant, du caractère révisable de cellesci; mais il ne défend pas pour autant une position communautarienne, promouvant des valeurs morales exclusives, un mode de vie unique autour d'un

31. Ce que notait également Kymlicka (2001): «Tout tend à démontrer que les membres de cultures libérales accordent [...] de l'importance à leur appartenance culturelle. Bien loin de supplanter l'identité nationale, la libéralisation s'est accompagnée d'un plus vif sentiment d'attachement à la nation ", p. 131.

32. Ibid, p. 21.

33. Dworkin (1985), p. 230-233; c'est à Kymlicka (1989) que l'on doit cette distinction entre la "structure de la culture " (cultural structure), au sens d'un "contexte de choix ", et le "caractère de la culture " (character of the culture), au sens de valeurs métaphysiques au sens ralwsien, des valeurs fondatrices dont la disparition ou la modification entrainerait une dépréciation de la culture en question, p. 166-167. 
telos unificateur ${ }^{34}$. Dans cette manière conciliatrice et nuancée de voir les choses, la nation ne peut être considérée que comme une "communauté imaginaire ", au sens où elle est le produit d'un pouvoir mental qui "permet à ses membres de se concevoir consciemment comme distincts des membres d'autres groupes ", et non une réalité substantielle, substrat de valeurs communes qu'on pourrait nommer «identité nationale ${ }^{35}$. Mais ce qui en fait aussi objectivement une entité imaginaire, c'est sa taille: la nation est par définition une vaste étendue. Or l'on accordera aisément que plus les structures sont importantes, plus l'anonymat grandit, de sorte que ce n'est plus que virtuellement que l'on peut se sentir appartenir à un même groupe ${ }^{36}$. Ce critère de la taille est fondamental, car il explique pourquoi l'idée de nation n'engendre pas l'enthousiasme des communautariens - dont on attendrait plutôt qu'ils la défendent en tant qu'elle promeut une certaine idée d'un bien commun fédérateur - et donne à voir, a contrario, sa pertinence dans l'appréhension du libéralisme très particulier de Rawls, ni authentiquement procédural ni totalement essentialiste.

En effet, cette idée d'un telos autour duquel tous se retrouvent ne peut s'incarner qu'au sein de petites structures, à un niveau proprement local. Les communautariens le reconnaissent tout à fait, à l'image d'un Sandel pour qui « la nation s'est avérée être un cadre trop vaste pour cultiver cette commune compréhension de soi nécessaire à une communauté constitutive ou formative ${ }^{37}$. En ce sens, la nation ne peut être, objectivement, que libérale : alors sa définition même exclut la possibilité d'une "politique du bien commun », sa « réalité virtuelle» fait que ses membres se sentent les héritiers d'un même

34. C'est ce qu'affirme Kymlicka (2001), commentant la vision rawlsienne de l'appartenance culturelle et retrouvant l'idée d'une identité mixte, faite de découvertes et de choix : "La liberté que les libéraux veulent conférer aux individus n'est pas essentiellement la liberté de s'émanciper de leurs propres langue et histoire, mais consiste plutôt en la liberté de se mouvoir au sein de leur culture sociétale, de se distancier des rôles culturels particuliers, de déterminer les caractéristiques particulières de la culture qui valent la peine d'être développées et celles dont on peut se détourner ", p. 134.

35 Tamir, ibid., p. 8.

36. Ici, toutefois, Tamir se démarque de Rawls dans la mesure où elle considère la nation comme distincte du peuple, logeant celui-ci à la même enseigne que la tribu ou la famille, à savoir un groupe dont il est, selon elle, relativement aisé de définir les frontières et les limites (p. 8); elle ajoute (ce qui achève de les séparer) que le peuple "a une existence indépendante de la conscience qu'en ont ses membres ", p. 65. Notre présent propos ne prend pas acte de cette distinction, d'autant que Tamir affirme par ailleurs qu'une nation doit pouvoir faire la démonstration «d'un certain nombre de caractères objectifs communs, comme la langue, l'histoire et le territoire, ainsi que la conscience de sa différence « (p. 66), ce qui n'est pas autre chose que la définition que Rawls donne du peuple. Pour une étude intéressante de l'idée de nation (autour de sa différenciation d'avec la notion d'ethnie [p. 28-29], du refus de l'homogénéité culturelle comme unique critère constitutif de la nation et de la nécessité, ce faisant d'une "communauté de citoyens « partageant "l'idée qu'il existe un domaine politique indépendant des intérêts particuliers et qu'ils doivent respecter son fonctionnement « [p. 44]), voir Schnapper (1994).

37. Sandel (1997), p. 272. 
lot de souvenirs, d'une même langue, d'un même esprit institutionnel, autant de valeurs qui constituent certes le terrain de jeu dans lequel ils ont à vivre mais qu'ils peuvent agencer à leur manière, créant leurs propres normes ${ }^{38}$. Comme l'écrit Kymlicka, montrant que l'idée nationale ne saurait être communautarienne parce que foncièrement non fondatrice: "Les mêmes membres d'une nation [...] ont en commun une langue et une histoire, mais sont souvent fondamentalement en désaccord sur ce qui a trait aux fins ultimes de la vie. L'identité nationale commune n'est pas, par conséquent, la base que requiert une politique communautarienne » (2001, p. 136).

La lecture de l'ensemble de l'œuvre rawlsienne nous permet d'asseoir définitivement cette idée d'une identité à mi-chemin entre un pur procéduralisme et un essentialisme avéré, au-delà d'une définition objective du concept de nation. C'est ce que donnent notamment à voir son rejet du cosmopolitisme ainsi que, d'une certaine manière, sa disqualification de l'immigration, acceptée mais non cautionnée.

En effet, à la lumière de la définition rawlsienne du peuple, il apparaît clairement que les membres d'un peuple bien ordonné sont unis par des liens très particuliers et très forts qui commandent eux-mêmes des obligations spécifiques et incommensurables, de sorte qu'on ne saurait en aucune manière comparer la relation qui nous unit, en tant que peuple déterminé, aux autres peuples bien ordonnés (quelles que soient les affinités qui peuvent par ailleurs nous rapprocher), aux rapports que nous entretenons les uns avec les autres en tant que membres d'une même nation ${ }^{39}$. Comme l'affirme Tamir, "vivre dans une communauté dont les membres partagent un sentiment «imaginaire» d'êtreensemble crée des responsabilités mutuelles » et, par conséquent, uniques (1999, p. 85). Il y a, de fait, une limite claire au devoir d'assistance aux peuples en difficulté; en d'autres termes, le droit des peuples ne saurait contenir l'obligation de veiller à appliquer un quelconque principe de différence au sein des sociétés que l'on accompagne sur le chemin de la décence. S'agissant du devoir d'aide :

son but est d'aider les sociétés entravées à gérer rationnellement et raisonnablement leurs propres affaires et à finir par devenir des membres de la Société de peuples bien ordonnés. Cela définit l'objectif de l'aide. Lorsqu'il est atteint, aucune aide supplémentaire n'est requise, même si la société à présent bien ordonnée peut demeurer relativement pauvre (DP, p. 136) ${ }^{40}$.

La position rawlsienne en la matière contraste, naturellement, avec la vision cosmopolitique pour qui une meilleure redistribution globale doit être préférée à une moins bonne: le bien-être des individus à l'intérieur d'une société

38. Sur l'idée que l'identité nationale doit se comprendre en dehors de la sphère normative, voir Tamir, ibid., p. 90.

39. Sur cette idée, voir Pettit (2006), p. 50.

40. Tamir semble défendre un position similaire lorsqu'elle affirme que "J'ai une obligation plus importante envers eux [les personnes défavorisées de ma ville] qu'envers les étrangers parce qu'ils sont membres de ma communauté $\phi$. (1993, p. 100-01; c'est Tamir qui souligne). 
donnée est tout aussi important que l'instauration d'institutions locales justes $^{41}$. En d'autres termes, le cosmopolitisme pose que le devoir d'aide ne saurait cesser avec la création desdites structures, tandis que la conception rawlsienne est indifférente au principe même d'une "décence matérielle ", conjuguant charité et solidarité exclusivement au niveau national. Pour reprendre la distinction que fait Grotius, par exemple, et que reprend Chauvier entre " devoir de justice » et "devoir d'humanité », le libéralisme politique global de Rawls est exclusivement concerné par le premier type de devoir - un «devoir de droit », dirait Kant - tandis que le cosmopolitisme ne saurait être imperméable, par ailleurs, au second type de devoir, un devoir de charitét2 Nous pourrions résumer cela sous la forme d'un paradoxe éclairant : en tant que membres d'un même peuple, nous devons obéir à un impératif hypothétique et faire de la charité, mobile par essence hétéronome, la maxime de notre action, tandis que les questions de justice internationale nous font renouer avec un kantisme épuré.

Venons-en à présent au problème de l'immigration. Dès TJ, Rawls identifiait la société politique à une société fermée, la définissant comme une «communauté nationale indépendante", autant dire un État-nation ${ }^{43}$. Il reprend par la suite cette définition et la complète, y ajoutant l'autosuffisance, la reproduction générationnelle pérenne, le contrôle d'un territoire défini comme autant de caractéristiques fondamentales, tout en thématisant, pour la première fois, la notion de fermeture comme l'absence de circulation intersociétale, ce qui exclut, comme de juste, tout mouvement migratoire ${ }^{44}$. La position rawlsienne gagne en cohérence lors du passage aux questions de justice

41. Nous nous en tenons à cette distinction, qui est aussi celle que fait Rawls (il est intéressant de noter combien celui-ci est lapidaire, s'agissant de confronter son droit des peuples à la théorie cosmopolitique). Notre propos n'est pas de discuter ici de la pertinence de l'une et l'autre conceptions de la justice internationale. Pour une analyse de ce type, à la fois originale et pertinente, voir les regards croisés de Kok-Chor Tan (2007), pour qui les nationalistes libéraux ont toutes les raisons d'être des internationalistes égalitaires, et de Leif Wenar (2006), qui récuse cette thèse.

42. Il est important de préciser ici que Chauvier (2006, p. 89-91) partage la thèse de Rawls selon laquelle on ne peut élargir le principe de différence à l'échelle globale, même s'il en donne une justification fort différente (notamment dans la distinction qu'il opère entre justice attributive et justice distributive). Par ailleurs, l'association libéralisme politique / devoir de justice, et cosmopolitisme / devoir d'humanité (de charité) n'est jamais explicitée en ces termes par Chauvier; ces deux alliances conceptuelles sont de notre fait.

43. TJ, p. 499. Sur cette idée, voir Seymour (1999).

44. «Une société bien ordonnée est une société qui se perpétue, une association autosuffisante d'êtres humains qui, comme un État-nation, contrôle un territoire donné. Ses membres voient leur communauté s'étendre dans le temps tout au long des générations successives et ils cherchent à se reproduire eux-mêmes ainsi que leur vie sociale et culturelle de manière quasi perpétuelle; c'est-à-dire qu'ils considéreraient comme inadmissible et étranger à la conception même de leur association le fait d'avoir à envisager une date pour y mettre un terme. Enfin, une société bien ordonnée est un système fermé; il n'existe pas de relations importantes avec d'autres sociétés, personne n'y entre de l'extérieur, car tous y sont nés et y vivent toute leur vie ", JD, p. 102. Voir également $L P$, p. 174 : «La société politique est close, il se trouve que nous en faisons partie et nous n'y entrons ni n'en partons volontairement, c'est même impossible. » 
globale, Rawls allant au terme de sa logique. Ainsi, puisque l'immigration est par principe impossible mais que nous ne pouvons, pratiquement, qu'en constater les effets désastreux en matière de déracinement et de perte de soi, éluder la difficulté en affirmant seulement que la société bien ordonnée est une société fermée ne saurait suffire. Il faut en effet travailler à l'éradiquer, et c'est ce à quoi la théorie rawlsienne s'emploie : « le problème de l'immigration n'est donc pas simplement mis de côté : en tant que problème grave, il est éliminé dans une utopie réaliste» $(D P$, p. 22).

Ce n'est pas là un discours anodin, mâtiné d'une certaine naïveté : il ne s'agit pas simplement de limiter les mouvements migratoires vers les sociétés libérales ordonnées, mais, à terme, d'en venir définitivement à bout ${ }^{45}$. Derrière cette prise de position pour le moins catégorique émerge l'idée qu'il y a quelque chose de fondamental, voire de sacré, à préserver de tout élément hétérogène - c'est ce qui explique qu'un simple contrôle ne suffit pas - et qui s'apparenterait à une sorte d'authenticité. Nul étonnement dès lors si Rawls affirme très nettement que le gouvernement, en tant qu' "agent efficace du peuple» doit "prend[re] la responsabilité de son territoire et de la taille de sa population, ainsi que du maintien de l'intégrité de son environnement » $(D P$, p. 21; l'italique est de nous), et que cette mission n'est ni provisoire ni ponctuelle, mais essentiellement pérenne ${ }^{46}$. Les mots employés par le philosophe sont très forts : ils donnent à penser que la nation est un reliquaire dont il faut veiller à prendre le plus grand soin, et le gouvernement un prêtre du culte qui officie tout à la fois avec abnégation, zèle et sens du devoir. Nous sommes en présence d'un credo substantiel dont les accents occultent parfois les professions de foi procédurales de Rawls.

En somme, et s'agissant particulièrement de ce dernier, ceux qui parlent d'un nationalisme libéral ne cherchent donc pas à réconcilier l'irréconciliable à tout prix, mais bien à rétablir une vérité qui ressemble fort à une tautologie. Chez le philosophe américain, la communauté politique se comprend comme une communauté nationale distincte de tout autre groupe, avec une somme de valeurs et d'attaches qui, loin d'emprisonner ses membres ou de geler leur capacité d'auto-détermination, leur offre un cadre spécifique où ils se construisent (et se déconstruisent) tout au long de leur existence. Mais une telle appartenance n'offre pas que des droits : elle engage et oblige en commandant des devoirs particuliers et particularistes.

Il est difficile, néanmoins, de passer outre une objection fondamentale faite au libéralisme procédural. Dans sa critique, Taylor, on l'a vu, mettait en

45. En s'attaquant, affirme Rawls, à ses causes principales, à savoir la persécution des minorités ethniques et religieuses, l'oppression politique sous toutes ses formes, ainsi que la pression démographique au sein du territoire national.

46. "La condition de pérennité est essentielle », DP, p. 21. La notion de « survivance ", plus connotée, recoupe partiellement cette idée d'aspiration à œuvrer pour préserver une culture à travers le temps, voir par exemple Taylor (1994), p. 83. 
avant l'idée selon laquelle celui-ci ne "prend pas au sérieux » les doctrines métaphysiques des individus et des groupes, les excluant de la sphère privée. Le philosophe canadien prenait, pour mémoire, l'exemple de la prière à l'école, montrant comment les libéraux procéduraux argumentaient leur refus d'inclure le culte (chrétien, en l'occurrence) à l'intérieur de l'enceinte scolaire. Selon lui, deux stratégies argumentatives étaient possibles. Un refus compréhensif, d'abord, témoignant du respect de l'autre et de sa différence, dans lequel on met l'interlocuteur face à l'iniquité de permettre l'exercice d'un culte déterminé tout en opposant une fin de non-recevoir à tous les autres; un refus méprisant, ensuite, dans lequel le vis-à-vis est confronté à son ignorance des principes moraux fondamentaux qui soutiennent la constitution américaine et au nom desquels on ne peut malmener la laïcité. S'ajoute à cela l'idée, comme on vient de le montrer dans la section précédente, que la communauté nationale est considérée comme étant ethniquement homogène ${ }^{47}$; le libéralisme politique que Rawls appelle de ses vœux semble ainsi s'éloigner durablement.

Commençons par le second élément. Il est important de garder à l'esprit que Rawls travaille sur des modèles simplifiés, voire ultra-simplifiés, et qu'il se situe, ce faisant, au niveau de la théorie idéale. Le philosophe américain est tout à fait conscient que la réalité des sociétés démocratiques libérales est tout autre que celle qu'il décrit; mais outre qu'une telle simplification n'enlève rien au fait que le philosophe américain dit bien ce qu'il dit, elle doit être replacée dans son contexte, un contexte très particulier dans lequel le pragmatisme joue un rôle essentiel:

En première approximation, la raison en est que le problème de la justice sociale concerne la structure de base en tant qu'elle constitue un contexte social et un système fermés. Commencer par la Société des Nations ne ferait que repousser d'un cran la tâche de trouver une théorie de la justice pour le contexte social. Il doit exister à un certain niveau un système fermé, et c'est l'objet pour lequel nous voulons une théorie. Nous sommes mieux préparés à traiter ce problème pour une société (cas illustré par une nation) conçue comme un système de coopération sociale plus ou moins autosuffisant et possédant une culture plus ou moins complète $(L P$, p. 325, n. 2$)$.

Ainsi, c'est parce qu'il est plus aisé de réfléchir sur une réalité aux contours bien délimités, quitte à la figer artificiellement, que Rawls travaille sur une société fermée, socialement autarcique, et avec une culture relativement homogène - et non pour des considérations métaphysiques. Il s'agit d'une hypothèse de travail qui pourra être élargie au problème de la justice entre les nations, si elle parvient à faire ses preuves en matière de cohérence et de stabilité $^{48}$.

47. Sur ce point, nous suivons l'analyse de Seymour (1999).

48. Ibid. 


\section{Comment prendre l'autre au sérieux?}

Venons-en à présent à la position de Taylor explicitée plus haut. Ce qui frappe à la lecture du propos du philosophe canadien, c'est sa prise de position immédiate. En effet, selon lui, le libéralisme procédural — précisément en vertu de la neutralité des pouvoirs publics sur lesquels il se fonde ainsi que sur la séparation tranchée entre public et privé - ne peut d'emblée que refuser l'idée de la prière à l'école. Or le libéralisme procédural n'est pas aussi dogmatique que Taylor veut bien le laisser entendre. De fait, Rawls fait montre d'une réelle ouverture d'esprit en la matière, au demeurant sur ce même sujet de la prière à l'école :

On pourrait penser qu'une position libérale consisterait à refuser qu'elle [la prière] soit permise dans les écoles publiques. Mais pourquoi donc? Il nous faut envisager toutes les valeurs politiques susceptibles d'être invoquées pour régler la question, et examiner de quel côté les raisons décisives font pencher la balance (IRPR, p. 195 ; italique ajouté).

Ainsi, pour le libéralisme politique, toute option est acceptable à condition de pouvoir la justifier par des raisons proprement politiques, qui puisent dans le fond commun de la raison publique, c'est-à-dire dans les idéaux implicites dans la culture démocratique d'un pays: tel est le «devoir de civilité » rawlsien. En ce sens, un laïc devra argumenter sa position avec la même rigueur que le détenteur d'une doctrine métaphysique religieuse, car la laïcité, bien que non religieuse, est elle aussi une doctrine compréhensive : il ne faut pas confondre « séculier» et «politique» (ibid., p. 172). Ce n'est donc pas sur la nature du positionnement des uns et des autres qu'il convient ici de s'arrêter (pour ou contre la prière à l'école), mais bien plutôt sur la nature des raisons invoquées à l'appui de celui-ci (raisons communes, politiques, ou raisons compréhensives). Ainsi, et contrairement à ce que croit Taylor, rien n'interdit à un libéral procédural de faire l'apologie publique de la prière à l'école, pourvu qu'il le fasse en des termes politiques ${ }^{49}$. C'est précisément là le sens qu'il faut donner à l'étonnement dont Rawls fait preuve face à ceux qui confondent forme et fond, reléguant d'emblée le libéralisme à la sphère de la laïcité et de la neutralité.

Mettre l'accent sur les raisons invoquées plutôt que sur la substance du propos n'équivaut en rien, comme auraient tendance à le croire certains, à être indifférent à la position défendue par son vis-à-vis, qui entérine d'office la

49. Cette question de l'interpénétration des discours faisant qu'il ne saurait plus y avoir de position déterminée qui serait défendue par une tradition ou un courant particuliers, mais que l'on assiste bien plutôt à un métissage discursif et à une circulation inter-paradigmatique, a été développée de manière fort pertinente, s'agissant des arguments conservateurs et libéraux pour (ou contre) l'avortement et l'homosexualité, par Sandel (2005), p. 122. Voir aussi, par l'auteur de ces lignes, "Paradigme libéral, paradigme républicain : du conflit à la composition interparadigmatique ", Revue philosophique de Louvain, 2008, à paraître. 
conclusion si l'argument est valable. Deux raisons essentielles peuvent être invoquées à cela. La première tient au fait qu'il faut bien distinguer entre le moment de la justification publique d'une position donnée lors d'un conflit ou d'une polémique opposant deux ou plusieurs parties (c'est-à-dire un moment ponctuel), du reste du temps où les doctrines compréhensives des uns et des autres coexistent normalement au sein de ce que Rawls nomme « la culture politique publique étendue» (IRPR, section 4, p. 182-186). Pour entrer dans le débat public, toute doctrine métaphysique doit satisfaire à une condition sine qua non, consistant dans la disposition à "présenter des raisons politiques appropriées » (ibid., p. 182). Dès l'instant où cette exigence est satisfaite se manifeste alors l'envie de connaître ou d'approfondir sa connaissance de ces doctrines métaphysiques qui acceptent de jouer le jeu politique. Le devoir de civilité a besoin de cette garantie pour s'exercer, et c'est seulement lorsque l'on a brisé la méfiance initiale, synonyme au mieux d'indifférence, au pire de rejet, que naît la disposition à s'ouvrir à l'autre. Les données du problème se trouvent de ce fait inversées, puisque c'est la satisfaction de la condition susmentionnée - se plier à l'injonction de présenter des raisons politiques fondant une prise de position donnée - qui annihile la peur d'être agressé par notre vis-à-vis. Rawls s'appuie ici sur une psychologie extrêmement réaliste :

De cette façon [lorsque tout un chacun prend la peine de rechercher des raisons politiques pour justifier son opinion], les citoyens attachés à des doctrines différentes sont rassurés et les liens de l'amitié civile en sortent renforcés (IRPR, 185-186).

La seconde raison intéresse la spécificité du devoir de civilité préconisé par Rawls. Y transparaît la volonté de respecter son interlocuteur, si chère à Taylor, et non d'être indifférent à son égard. En effet, les critiques de Rawls ont tendance à oublier la réciprocité qui conduit débats et controverses, et dont le principe récuse l'existence d'une position de prime abord déjà légitime. Pareil statut ne s'acquiert que par la capacité à présenter des raisons authentiquement politiques à l'appui d'une opinion donnée, de sorte que le respect que je dois à autrui se lit dans la volonté qui m'anime de me mettre au même niveau que lui : nous sommes à égalité dans la discussion qui nous réunit parce que chacun d'entre nous doit travailler à rendre sa position acceptable aux yeux de l'autre. D'un autre côté, respecter l'autre, ce n'est ni lui dire ce qu'il a envie d'entendre ni lui permettre de se justifier en usant des arguments qu'il emploierait s'il voulait se convaincre lui-même. Face à cette optique démagogique visant à désamorcer d'emblée les tensions inhérentes à toute polémique qui mettrait en scène des questions épineuses et des sensibilités différentes, le 
libéralisme politique de Rawls invite à l'exigence vis-à-vis de soi et des autres $^{50}$.

$$
* * *
$$

Manifestement, nulle part, dans la pensée du philosophe américain, ne transparaît mieux le respect dû aux autres que dans le traitement qu'il réserve à la question de savoir s'il faut tolérer les intolérants. La réponse commune, celle qui réunit le plus de suffrage est bien évidemment la réponse négative. Lidée implicite qui sous-tend cette dernière est que les intolérants ne méritent pas d'être tolérés, car ils font eux-mêmes preuve d'intolérance à l'égard d'autrui ${ }^{51}$.

Or Rawls récuse ce raisonnement. Il reformule les données du problème de sorte que le critère de la tolérance ne soit pas envisagé en termes de mérite, mais en termes de "raison suffisante » : un comportement intolérant constitue-t-il une raison suffisante pour priver de liberté celui qui en est l'auteur ? La réponse de Rawls est non, à une exception près : c'est uniquement quand nos "intérêts légitimes" sont en jeu qu'il faut être intolérant envers ceux qui le sont ${ }^{52}$. Cela signifie, d'une part, que si le comportement intolérant d'autrui ne constitue pas une menace immédiate pour notre vie, notre sécurité et notre liberté, alors nous n'avons aucune raison d'agir contre lui; cela signifie, d'autre part, que la suspicion d'intolérance ne peut en aucun cas être un motif valable pour empêcher celui qui en est l'objet de jouir de sa liberté.

50. Le libéralisme de Rawls, ainsi conçu, répond adéquatement à ce que Walzer (1997b) appelle de ses vœux, à savoir le fait de «retrouver une citoyenneté active " (p. 176), dans laquelle chacun a la "possibilité » (p. 180) d'être un citoyen vertueux, et pas simplement un égoïste motivé «par des amitiés mercantiles ». De fait, Walzer affirme très justement que la question n'est plus celle du «primat » de la citoyenneté active, mais celle de sa «possibilité »; en ce sens, le devoir de civilité rawlsien, l'introduction des doctrines métaphysiques au sein du débat public pour que tous puissent avoir accès à la discussion, la propension naturelle à cultiver le sens de la justice pour lui-même, la promotion et le souci de bâtir des institutions justes pour elles-mêmes, l'amitié civile plutôt que "l'amitié mercantile ", tout cela en contribuant à créer ce "nous moral que nous sommes" (Larmore, 2000, p. 219), explique comment cette collection d'individus différents qui constitue un peuple peut rester soudée malgré le "fait du pluralisme ", c'est-à-dire malgré l'institutionnalisation en son sein du désaccord moral, politique et philosophique. Sur ce point précis, Rawls rejoint Taylor, 2004, p. 189 passim.

51. Ce à quoi souscrit Walzer (1998), jusqu'à une certaine limite: les intolérants doivent être tolérés de manière "partielle ». En effet, si leur intolérance est "une bonne raison de leur refuser le pouvoir politique », elle ne saurait être une raison valable pour restreindre leur liberté par ailleurs, p. 118. Le philosophe américain fonde son raisonnement sur l'idée selon laquelle c'est la séparation entre l'Église et l'État qui motive légitimement un comportement d'intolérance envers les intolérants, et elle seule. Le raisonnement walzerien rejoint celui de Rawls, à une différence près, qui n'est pas des moindres. Pour l'auteur de $T J$, la laïcité ne bénéficie pas d'un préjugé favorable et ne constitue pas l'idéal vers lequel la société bien ordonnée doit tendre, car elle est, comme nous l'avons vu, une doctrine métaphysique. En ce sens, elle ne saurait commander aucune décision politique, a fortiori une décision institutionnalisant un comportement intolérant.

52. «Une condition plus rigoureuse est nécessaire: nos intérêts légitimes doivent être sérieusement menacés », TJ, p. 255. 
Pour être prise en considération, l'intolérance doit être effective et se manifester concrètement.

La perspective de Rawls est motivée, comme de juste, par un kantisme intransigeant: le Bien, parce qu'il est relatif, ne peut jamais constituer une norme valide pour l'action. Or en jugeant l'intolérance dont font preuve les intolérants alors que leur comportement ne nous lèse pas, il y a un risque que notre jugement soit un jugement de valeur. Il y a un risque que notre jugement se fasse à partir de notre idée de ce qu'est le Bien et la vie bonne. Mais qui peut prétendre que sa propre conception du bien est la seule valable, qu'elle est universelle? En jugeant l'intolérant, il y a donc le risque que nous fassions nous-mêmes preuve d'intolérance. Comme l'explique Rawls, ce n'est pas parce que tel individu fait l'objet d'une suspicion d'intolérance (voire agit de manière intolérante), que cela nous autorise nous-mêmes à être intolérants. Bien au contraire, "puisqu'une juste constitution existe, tous les citoyens ont un devoir naturel de justice de la soutenir. Nous ne sommes pas dégagés de ce devoir chaque fois que les autres sont disposés à agir injustement» (TJ, p. 255; l'italique est de nous).

Les limites du principe de tolérance rawlsien, dira-t-on toutefois, sont claires. En effet, déterminer que nos intérêts légitimes sont menacés n'est pas chose facile. Le passage de l'absence de danger à la menace est parfois très rapide, et réellement difficile à prévoir. La question qui se pose alors est celle de la nécessité du "principe de précaution » en vertu duquel "mieux vaut prévenir que guérir »: il est peut-être fortement recommandé de ne pas tolérer les intolérants pour éviter qu'un jour nous ne soyons directement menacés par eux, au pied du mur, sans recours possible.

Toute la question serait alors de savoir comment exercer notre intolérance pour éviter de commettre une injustice envers la personne jugée fanatique et sectaire. Est-ce seulement possible ? La grande leçon de Rawls est de montrer que la liberté est trop précieuse pour engager ce type de calcul dont le simple bon sens nous invite à penser que la démocratie ne sortirait pas indemne.

\section{Références}

Allen, A. \& M. C. Reagan (dir.). Debating Democracy's discontent. New York, Oxford University Press, 1998.

Berlin, Isaiah. À contre courant. Essai sur l'histoire des idées, trad. A. Berelowitch, Paris, Albin Michel, 1988.

Berten, A., H. Pourtois, P. Da Silveira (dir.), Libéraux et communautariens. Paris, Presses Universitaires de France, 1997.

Bouvier, Pierre. Le lien social, Paris, Gallimard, 2005.

Chauvier, Stéphane. Justice et droits à l'échelle globale, Paris, Vrin/Ehess, 2006.

Dworkin, Ronald. A Matter of Principle, London, Harvard University Press, 1985.

Forst, Rainer. «How not to Speak about Identity : the Concept of the Person in A Theory of Justice ", Philosophy and Social Criticism, 18, 1992.

Kymlicka, Will. Liberalism, Community and Culture, Oxford University Press, 1989. 
- La citoyenneté multiculturelle. Une théorie libérale du droit des minorités, trad. Patrick Savidan, Paris, La Découverte, 2001.

Laforest, G et P. Lara (dir.). Charles Taylor et l'interprétation de l'identité moderne. Paris, Cerf, 1998.

Larmore, Charles. "Le «nous" moral que nous sommes", in Comprendre: les identités culturelles, sous la direction de Will Kymlicka et Sylvie Mesure, $\mathrm{n}^{\circ} 1$, Paris, PUF, 2000, pp. 219-234.

Martin, R. et D. A. Reidy (dir.). Rawls's Law of Peoples. A Realistic Utopia. Blackwell Publishing, 2006.

Mestiri, Soumaya. De l'individu au citoyen. Rawls et le problème de la personne, Éditions de la Maison des sciences de l'homme, "Philia-Monde ", 2008a.

__. «Paradigme libéral, paradigme républicain: du conflit à la composition inter-paradigmatique ", Revue philosophique de Louvain, 2008b, à paraître.

Mill, John Stuart. De la liberté, trad. L. Lenglet d'après la traduction de Dupond-White, Paris, Gallimard, Folio Essais, 1990.

Pettit, Philip. "Rawls's Peoples ", in Rawls's Law of Peoples, 2006, p. 38-56.

Rawls, John [TJ]. Théorie de la justice, trad. C. Audard, Paris, Seuil, 1986.

—. "Le constructivisme kantien dans la théorie morale ", in Justice et démocratie [JD], Paris, Seuil, 1993.

. [LP]. Libéralisme politique, trad. C. Audard, Paris, PUF, 1995.

. [PL]. Political Liberalism, New York, Columbia University Press, 1996.

. [DJP]. DJP = Débat sur la justice politique, avec J. Habermas, Cerf, «Humanités ", 1997.

- [SJ] (1963) «The Sense of Justice », in Collected Papers, Samuel Freeman (ed.), Cambridge, Harvard University Press, 1999a, pp. 117-129.

. [LODFP] (1964). «Legal Obligation and the Duty of Fair Play », in Collected Papers, Samuel Freeman (ed.), Cambridge, Harvard University Press, 1999b, pp. 117-129.

. [IRPR]. «L'idée de raison publique reconsidérée », in Paix et démocratie, trad.

B. Guillarme, Paris, La Découverte, 2006a.

- [DP]. «Le Droit des peuples », in Paix et démocratie, trad. B. Guillarme, Paris, La Découverte, 2006b.

Roman, Jœl. "Le pluralisme de Michael Walzer », in Pluralisme et démocratie, Paris, Éditions Esprit, 1997, pp. 7-28.

Sandel, Michael. "La république procédurale et le moi désengagé ", in Libéraux et communautariens, 1997.

. Democracy's Discontent. America in Search of a Public Philosophy, Harvard University Press, Ms, 1998.

- Public Philosophy. Essays on Morality in Politics, Harvard University Press, Ms, 2005.

Schnapper, Dominique. La communauté des citoyens. Sur l'idée moderne de nation, Paris, Gallimard, 1994.

Seymour, Michel. "Rawls et le droit des peuples ", Philosophiques, 26/1, printemps, 1999, pp. 109-137.

Skinner, Quentin. "The Idea of Negative Liberty: Philosophical and Historical Perspectives ", Philosophy in History, R. Rorty, J. B. Schneewind et Q. Skinner (dir.), Cambridge, Cambridge University Press, 1984, pp. 193-221.

Tamir, Yæl. Liberal Nationalism, Princeton University Press, Princeton, 1993. 
Tan, Kok-Chor. « Nationalisme libéral et internationalisme égalitaire », Philosophiques, printemps 2007, vol. 34, $\mathrm{n}^{\circ}$ 1, 2007.

Taylor, Charles. "La politique de la reconnaissance ", in Multiculturalisme. Différence et démocratie, Paris, Aubier, 1994.

"Quiproquos et malentendus: le débat comunautariens-libéraux », Berten, Pourtois, da Silveira (dir.), Paris, Presses Universitaires de France, 1997, pp. 87120.

—. "Living with Difference », in Debating Democracy's Discontent, A. Allen \& M. C. Reagan (dir.), New York, Oxford University Press, 1998,

-. "Quel principe d'identité collective? ", in L'Europe au soir du siècle. Identité et démocratie, Paris, Éditions Esprit, 1999, pp. 59-66.

- Modern Social Imaginaries, Dukes University Press, Durham and London, 2004.

Wenar, Leif. "Why Rawls Is Not A Cosmopolitan Egalitarian », in Rawls's Law of Peoples, Reidy and Martin (eds), 2006, pp. 95-114.

Walzer, Michael. "La critique communautarienne du libéralisme », trad. P. Destrée, in Pluralisme et démocratie, Paris, Éditions Esprit, 1997a, pp. 53-82.

- "Communauté, citoyenneté et jouissance de droits ", trad. J.-C. Monod, in Pluralisme et démocratie, Paris, Éditions Esprit, 1997b, pp. 167-181.

- Sphères de justice. Une défense du pluralisme et de l'égalité, Paris, Seuil, 1997c.

- Traité sur la tolérance, trad. Chaïm Hutner, Paris, Gallimard, 1998. 